\section{Leberkrebsinzidenz nach DAA-Behandlung bei 0,29 pro 100 Patientenjahre}

Die Patienten wurden in der Analyse im Schnitt 6,1 Jahre beobachtet. Bei 3.271 Patienten wurde wenigstens sechs Monate nach HCV-Therapiebeginn eine Leberkrebsdiagnose gestellt. Die Autoren berechneten folgende Inzidenzen (Fälle pro 100 Patientenjahre):

- 3,32 bei Zirrhotikern mit Therapieversagen

- 1,97 bei Zirrhotikern mit erfolgreicher Therapie

- 0,87 bei Patienten ohne Zirrhose und Therapieversagen

- 0,24 bei Patienten ohne Zirrhose und erfolgreicher Therapie
Bei HCV-Elimination zeigten sich folgende adjustierten Hazard Ratios in Abhängigkeit vor der Behandlung:

0,29 bei Therapie mit DAA

- 0,48 bei Therapie mit DAA plus Interferon

- 0,32 bei Therapie mit Interferon

Fazit: Die heutigen Interferon-freien Standardtherapien senken das Leberkrebsrisiko nach erfolgter HCV-Heilung um $71 \%$. Das Krebsrisiko liegt dabei nicht höher als bei einer erfolgreichen Interferon-Therapie.

Dr. med. Dirk Einecke

\title{
Studie untersucht Adhärenz: Wie gut wirkt die HCV-Therapie bei mäßiger Therapietreue?
}

\author{
Moderne Therapien heilen nahezu alle HCV-Patien- \\ ten. Eine Studie mit der Kombination Glecaprevir/ \\ Piprentasvir zeigt jetzt: Das gilt selbst für Patienten \\ mit Compliance-Problemen.
}

Bei Glecaprevir/Piprentasvir (G/P, Maviret ${ }^{\circledR}$ ) handelt es sich um eine kürzlich zugelassene, gegen alle sechs HCV-Genotypen wirksame Kombination für nicht vorbehandelte Patienten, berichtete Dr. Ashley S. Brown vom Imperial College in London. Patienten ohne Zirrhose werden acht Wochen behandelt, Patienten mit Zirrhose zwölf Wochen. Die dabei erzielten Heilungsoder SVR12-Raten liegen bei $98 \%$ respektive $96 \%$. Auch bei häufigen Resistenzpolymorphismen wie Y93H oder Q80K wirkt das duale Medikament zuverlässig.

Ziel der vorgestellten Studie war es, im Rahmen einer Analyse aller Phase-II- und Phase-III-Zulassungsstudien die Wirksamkeit in Abhängigkeit von der Therapieadhärenz zu untersuchen [Brown AS et al. AASLD 2017, Abstract 198). Letztere wurde mittels Pillenzählung bestimmt. Non-Adhärenz war definiert als Einnahme von $<80 \%$ oder $>120 \%$ der vorgesehenen Tabletten bei einer Kontrolluntersuchung. Von 2.091 untersuchten $\mathrm{Pa}$ tienten erwiesen sich 1.851 als adhärent, 240 Patienten wiesen Compliance-Probleme auf, wobei $235 \mathrm{zu}$ wenig und fünf zu viele Tabletten eingenommen hatten.

\section{Prädiktoren für schlechte Adhärenz}

Als Prädiktoren oder Risikofaktoren für Non-Adhärenz konnten in einer multivariaten Regressionsanalyse Alkohol- und Tabakkonsum ermittelt werden, zudem kompensierte Leberzirrhose und schwere Niereninsuffizienz sowie - überraschenderweise - Infektion durch den Genotyp 3.

\section{Medikamentenkombination verzeiht Einnahmefehler} Die nachlässige Tabletteneinnahme hatte jedoch keinen negativen Effekt auf das Behandlungsergebnis. Die SVR12-Raten betrugen in der ITT-Analyse $98 \%$ (Adhärenz) und $95 \%$ (Non-Adhärenz). Bei ausschließlicher Betrachtung der tatsächlichen virologischen Versager in der modifizierten ITT-Analyse beliefen sich die Heilungsraten in beiden Gruppen auf $99 \%$. Insgesamt kam es in beiden Gruppen zusammen bei neun Patienten zu einem virologischen Versagen und bei 19 Patienten $\mathrm{zu}$ einem Rückfall.

Nach Berechnungen der Autoren liegt die Heilungsrate unter einer G/P-Therapie mindestens bei $90 \%$, wenn nur die Hälfte aller Medikamente eingenommen wird. Die Autoren wollen diese Daten aber nicht als Aufforderung zum Pillensparen interpretiert wissen, sondern als eine Beruhigung für den Arzt und den Patienten, wenn es mit der Einnahmedisziplin zuweilen hapert.

Dr. med. Dirk Einecke

The Liver Meeting 2017, AASLD-Kongress, Washington, 20. - 24.10.2017

HCV-Ausheilung: Deutlicher Zuwachs der

Lebensqualität bei multimorbiden Patienten

Eine HCV-Ausheilung führt kurz- und mittelfristig zu einer Verbesserung der gesundheitsbezogenen Lebensqualität. Patienten mit Komorbiditäten profitieren am deutlichsten.

Drei-Jahresergebnisse bezüglich der Lebensqualität nach einer HCVTherapie berichtete Dr. Zobair Younoussi, Falls Church/Virginia [Younussi ZM et al., AALSD 2017, Abstract 64].

In der Studie füllten 3.486 Patienten halbjährlich eine Kurzform des "Health related Quality of Life"-Score (HRQL) aus, der 36 Parameter erfasste (SF-36). Die im Schnitt 53-jährigen Patienten wiesen zu $16 \%$ eine Zirrhose auf, zu 12\% eine HIV-Koinfektion, zu 10\% einen Diabetes mellitus, zu $25 \%$ eine Depression und zu $16 \%$ eine Angststörung. Der SR-36-Summary Score zeigte signifikante Verbesserungen sowohl für das körperliche (von 50,5 auf 52,5) als auch das mentale (von 49,5 auf 52) Wohlbefinden nach einem Jahr sowie nach drei Jahren.

In der multivariaten Analyse erwiesen sich Zirrhose, Depression, Angst und erhebliche Abgeschlagenheit als Faktoren, welche die Lebensqualität am stärksten beeinträchtigten. Patienten mit den Komorbiditäten Depression, Fatigue, Schlafstörungen, Diabetes mellitus und Zirrhose erzielten andererseits den größten Gewinn an Lebensqualität durch die Heilung der Hepatitis $C$. Dr. med. Dirk Einecke

The Liver Meeting 2017, AASLD-Kongress, Washington, 20. -24.10 .2017$ 